\title{
Reference Values for 27 Clinical Chemistry Tests in 70-Year-Old Males and Females
}

\author{
Lena Carlsson $^{\mathrm{a}}$ Lars Lind $^{\mathrm{b}}$ Anders Larsson ${ }^{\mathrm{a}}$ \\ Department of Medical Sciences, ${ }^{a}$ Clinical Chemistry and ${ }^{b}$ Internal Medicine, Uppsala University, Uppsala, Sweden
}

\section{Key Words}

70-year-old population $\cdot$ Reference values $\cdot$ Biological markers

\begin{abstract}
Background: Reference values are usually defined based on blood samples from healthy men or nonpregnant women in the age range of 20-50 years. These values are not optimal for elderly patients, as many biological markers change over time and adequate reference values are important for correct clinical decisions. Objective: To validate NORIP (Nordic Reference Interval Project) reference values in a 70-year-old population. Methods: We studied 27 frequently used laboratory tests. The 2.5th and 97.5th percentiles for these markers were calculated according to the recommendations of the International Federation of Clinical Chemistry on the statistical treatment of reference values. Results: Reference values are reported for plasma alanine aminotransferase, albumin, alkaline phosphatase, pancreas amylase, apolipoprotein A1, apolipoprotein B, aspartate aminotransferase, bilirubin, calcium, chloride, cholesterol, creatinine, creatine kinase, C-reactive protein, glucose, $\gamma$-glutamyltransferase, $\mathrm{HDL}$-cholesterol, iron, lactate dehydrogenase, LDL-cholesterol, magnesium, phosphate, potassium, sodium, transferrin, triglycerides, urate and urea. Conclusion: Reference values calculated from the whole population and a subpopulation without cardiovascular disease showed strong concordance. Several of the reference interval limits were outside the $90 \% \mathrm{Cl}$ of a Scandinavian population (NORIP).
\end{abstract}

Copyright $\odot 2009$ S. Karger AG, Basel
(C) 2009 S. Karger AG, Basel

0304-324X/10/0563-0259\$26.00/0

Fax +4161306 1234

E-Mail karger@karger.ch

www.karger.com
Accessible online at: www.karger.com/ger

\section{Introduction}

Today, there are a limited number of instruments suited for high-throughput clinical use. Results from internal and external quality assessment programs confirm that there are acceptable interlaboratory agreements [1]. In principle it should be possible to have the same reference values, independent of which laboratory performs the measurements. There is currently a trend to establish common reference values where possible $[2,3]$. Increasing national and international communication between healthcare providers also strengthens the need for the harmonization of measurements and reference values in laboratories [4].

Presently, only a few clinical laboratories produce their own reference values, with the great majority using reference values from the literature [5]. The use of such reference values shows the need for publications that present reference values based on modern methods. Such publications should include not only the reference value, but also provide information regarding reference population, methods used, traceability and imprecision. Without such information, it is difficult to transfer and use published reference values in other laboratories. Unfortunately, many publications on reference values lack one or several of these important pieces of information.

Reference values are usually given for each sex, but may not take into account variation in reference values due to age. Many biomarkers change with age and, therefore, reference values based on a population in the age 
Table 1. Total analytical imprecision for the method used to calculate the reference values

\begin{tabular}{|c|c|c|}
\hline Analyte & Concentration & $\mathrm{CV}, \%$ \\
\hline \multirow[t]{2}{*}{ Alanine aminotransferase, $\mu \mathrm{kat} / \mathrm{l}$} & 0.76 & 2.1 \\
\hline & 1.9 & 1.9 \\
\hline \multirow[t]{2}{*}{ Albumin, g/l } & 32 & 0.3 \\
\hline & 49 & 0.4 \\
\hline \multirow[t]{2}{*}{ Alkaline phosphatase, $\mu$ kat/l } & 2.3 & 1.8 \\
\hline & 10.6 & 1.6 \\
\hline \multirow[t]{2}{*}{ Amylase (pancreas), $\mu$ kat/l } & 1 & 0.5 \\
\hline & 0.3 & 1.1 \\
\hline \multirow[t]{2}{*}{ Apolipoprotein A1, g/l } & 0.68 & 1.8 \\
\hline & 2.0 & 1.2 \\
\hline \multirow[t]{2}{*}{ Apolipoprotein B, g/l } & 0.47 & 4.5 \\
\hline & 1.68 & 2.4 \\
\hline \multirow[t]{2}{*}{ Bilirubin, $\mu \mathrm{mol} / 1$} & 10 & 2.5 \\
\hline & 26 & 2.7 \\
\hline \multirow[t]{2}{*}{ Calcium, mmol/l } & 2.2 & 0.9 \\
\hline & 2.9 & 1.2 \\
\hline \multirow[t]{2}{*}{ Chloride, $\mathrm{mmol} / \mathrm{l}$} & 85 & 0.7 \\
\hline & 108 & 0.5 \\
\hline \multirow[t]{2}{*}{ Cholesterol, mmol/l } & 5.7 & 0.5 \\
\hline & 7.8 & 0.6 \\
\hline \multirow[t]{2}{*}{ Creatinine, $\mu \mathrm{mol} / \mathrm{l}$} & 70 & 4.8 \\
\hline & 94 & 4.8 \\
\hline \multirow[t]{2}{*}{ Creatinine kinase, $\mu$ kat/l } & 1.8 & 1.4 \\
\hline & 2.8 & 0.7 \\
\hline \multirow[t]{2}{*}{ C-reactive protein, mg/l } & 2.6 & 1.4 \\
\hline & 8 & 0.8 \\
\hline \multirow[t]{2}{*}{$\gamma$-Glutamyltransferase, $\mu$ kat/l } & 1.3 & 0.7 \\
\hline & 2.4 & 0.9 \\
\hline \multirow[t]{2}{*}{ HDL-cholesterol, mmol/l } & 0.9 & 2.3 \\
\hline & 1.2 & 2.1 \\
\hline \multirow[t]{2}{*}{ Iron, $\mu \mathrm{mol} / 1$} & 18 & 0.9 \\
\hline & 37 & 1.7 \\
\hline \multirow[t]{2}{*}{ Lactate dehydrogenase, $\mu$ kat/l } & 1.25 & 1.3 \\
\hline & 2.7 & 1.0 \\
\hline \multirow[t]{2}{*}{ LDL-cholesterol, mmol/l } & 1.6 & 1.0 \\
\hline & 2.8 & 1.1 \\
\hline \multirow[t]{2}{*}{ Magnesium, mmol/l } & 0.9 & 1.4 \\
\hline & 1.7 & 0.9 \\
\hline \multirow[t]{2}{*}{ Phosphate, mmol/l } & 1.1 & 0.6 \\
\hline & 1.7 & 0.5 \\
\hline \multirow[t]{2}{*}{ Potassium, mmol/l } & 3.3 & 1.5 \\
\hline & 5.5 & 1.0 \\
\hline Sodium, mmol/l & 114 & 0.8 \\
\hline & 141 & 0.6 \\
\hline Transferrin, g/l & 2.0 & 0.7 \\
\hline & 3.0 & 0.8 \\
\hline Triglycerides, mmol/l & 0.9 & 1.1 \\
\hline & 2.2 & 1.1 \\
\hline Urate, $\mu \mathrm{mol} / \mathrm{l}$ & 280 & 2.1 \\
\hline & 580 & 0.7 \\
\hline Urea, mmol/l & 5.3 & 0.6 \\
\hline & 11.1 & 0.6 \\
\hline
\end{tabular}

$\mathrm{CV}=$ Coefficient of variation. range of 20-50 years may not be appropriate for 70-yearold patients.

Clinical chemistry reference values for elderly persons are sparse and often intermixed with those of younger subjects. Elderly patients are often treated by the health care system, and they seek health care more often than those who are 20-50 years of age. The approximate median age of patients treated in most hospital wards is 70 years. Since appropriate reference values are essential for quality patient care, we felt an urge to set up reference values for our most widely used serum analy tes representative for our elderly patients. The criteria for defining a 'healthy' population for establishing reference values vary. If very strict criteria are used in an elderly population, the group may become very small and not very representative of the whole population. Many elderly individuals are on some type of medication and often have symptoms of cardiovascular disease (CVD). When we set up the selection criteria for this study, we decided to exclude all individuals with diabetes and then calculate reference values. The aim of the study was to validate reference values from the Nordic Reference Interval Project (NORIP) in a 70-year-old population.

\section{Subjects and Methods}

\section{Subjects}

All subjects aged 70 years living in Uppsala, Sweden, were eligible. The subjects were chosen from the community register and were invited to participate in a randomized order from the start of the study in April 2001 to the last included subject in June 2005. The subjects received an invitation by letter within 1 month of their 70th birthday in order to standardize for age. Of the 2,025 subjects invited, 1,016 subjects were investigated, resulting in a participation rate of $50.1 \%$. The study is part of the Prospective Investigation of the Vasculature in Uppsala Seniors (PIVUS) study [6].

The study was approved by the ethics committee of the University of Uppsala, and the participants gave their informed consent.

The participants were asked to answer a questionnaire about their medical history, smoking habits and regular medication. All subjects were investigated in the morning after an overnight fast. No medication or smoking was allowed after midnight. During the investigation, the subjects were supine in a quiet room maintained at a constant temperature. CVD was defined as myocardial infarction, stroke and heart failure, but not hypertension.

Blood glucose was analyzed in whole blood on a HemoCue

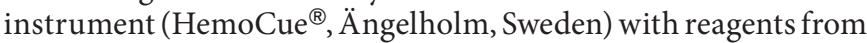
the manufacturer. Blood samples were collected in Vacutainer tubes without additives. Total cholesterol, HDL-cholesterol, LDLcholesterol and triglycerides were centrifuged at room temperature in a Genesis FE500 (Tecan, Männedorf, Switzerland) and analyzed on the day the samples were collected. Samples for the 
Table 2. Traceability of the standardizations of the methods used to calculate the reference values

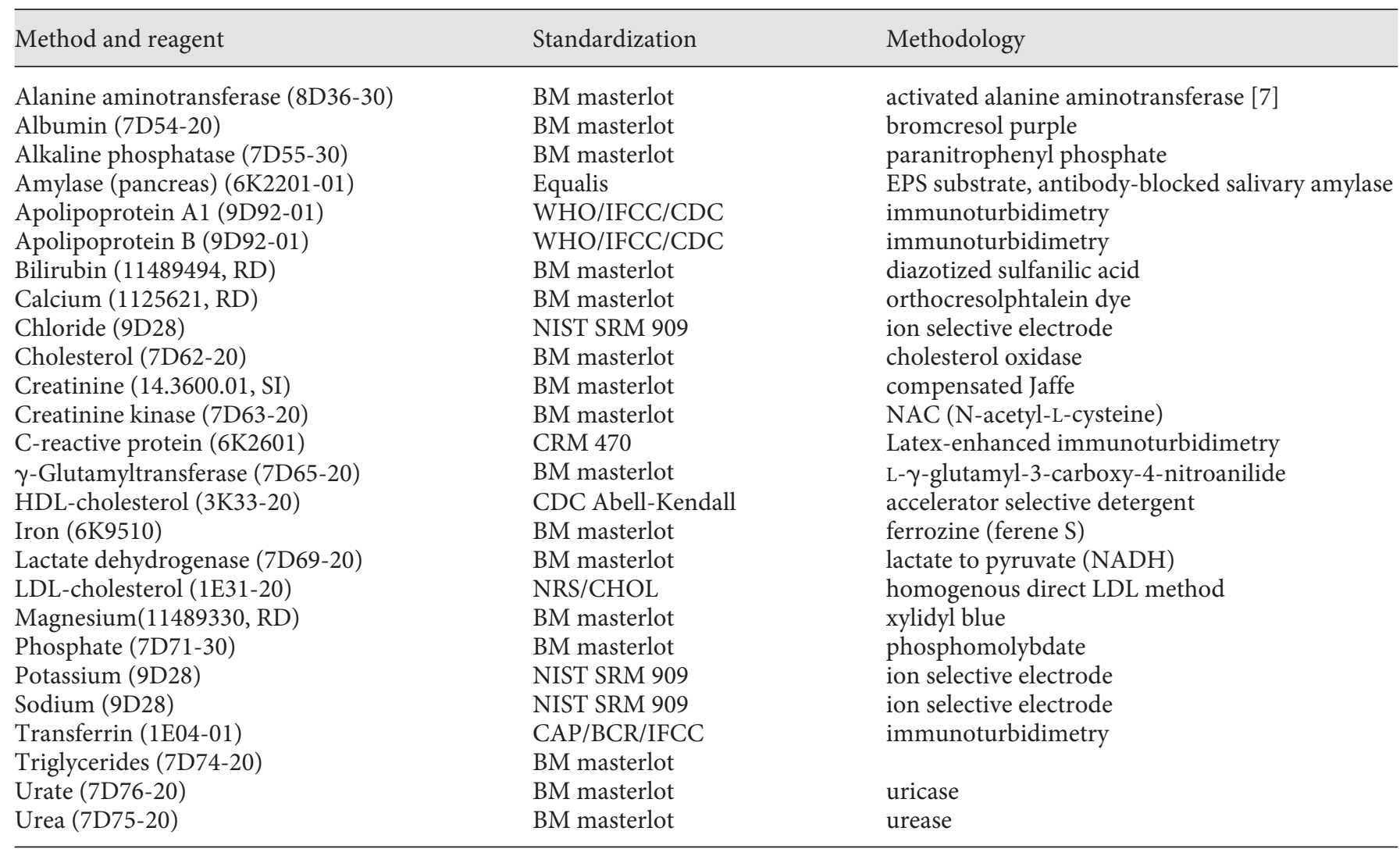

Analyte, reagents (within brackets), methodology and traceability of the standardizations of the methods used to calculate the reference intervals. If not stated otherwise, all reagents were from Abbott Laboratories. RD = Roche Diagnostics (Mannheim, Germany); SI = Synermed International (Westfield, Ind., USA).

other analytes were centrifuged in a refrigerated centrifuge and stored at $-80^{\circ} \mathrm{C}$ until analysis. Samples from individuals with a diagnosis of diabetes mellitus or a fasting plasma glucose $\geq 7.0$ $\mathrm{mmol} / \mathrm{l}$ were excluded from the study.

\section{Instrument}

All measurements were performed on an Architect Ci8200 analyzer (Abbott Laboratories, Abbott Park, Ill., USA) and reported using SI units. Total analytical imprecision of the assays is presented in table 1 . Traceability of the methods is presented in table 2 .

\section{The Nordic Reference Interval Project}

NORIP, established in March 1998 (www.furst.no/norip/), was initiated to establish common Nordic reference intervals for 25 of the most frequently requested analytes in clinical biochemistry [8]. The reference material is based on 3,035 reference individuals ( $47 \%$ males, $53 \%$ females). The majority of the individuals were of Nordic origin (96\%). The age distribution of the NORIP population was: $18-30$ years for $793,31-50$ years for $928,51-70$ years for 832 and $>70$ years for 449 subjects.

Clinical Chemistry Test Reference Values

in Elderly Populations

\section{Statistical Calculations}

Calculations of reference values were performed by bootstrap estimation, utilizing RefVal 4.0 (Department of Clinical Chemistry, Rikshospitalet, Oslo, Norway). RefVal fulfils the recommendations of the International Federation of Clinical Chemistry for the statistical treatment of reference values $[9,10]$. Comparisons between groups were performed with a Mann-Whitney U test (Statistica, StatSoft, Tulsa, Okla., USA). For comparisons between the reference values in NORIP and the reference intervals obtained in this study, 90\% confidence intervals were used.

\section{Results}

\section{Description of the Study Population}

Basic characteristics of the total samples based on the questionnaire are presented in table 3. Approximately $10 \%$ of the cohort reported a history of coronary heart disease, $4 \%$ reported stroke and 9\% diabetes mellitus. 
Table 3. Basic characteristics of the total sample $(n=1,016)$

\begin{tabular}{lc}
\hline Variable & Mean \pm SD \\
\hline Females, \% & 50.2 \\
Height, cm & $169 \pm 9.1$ \\
Weight, kg & $77 \pm 14$ \\
Waist circumference, cm & $91 \pm 12$ \\
BMI & $27.0 \pm 4.3$ \\
Waist/hip ratio & $0.90 \pm 0.075$ \\
SBP, mm Hg & $150 \pm 23$ \\
DBP, mm Hg & $79 \pm 10$ \\
Myocardial infarction, \% & 7.1 \\
Stroke, \% & 3.7 \\
Angina pectoris, \% & 8.1 \\
Congestive heart failure, \% & 3.8 \\
Diabetes, \% & 8.7 \\
Current smoking, \% & 11 \\
Any regular drug, \% & 70 \\
Any cardiovascular drug, \% & 45 \\
Any antihypertensive medication, \% & 32 \\
B-Blockers, \% & 22 \\
Calcium antagonists, \% & 11 \\
Diuretics, \% & 13 \\
ACE-inhibitors, \% & 8.5 \\
Angiotensin II blockers, \% & 8.3 \\
Digoxin, \% & 2.1 \\
Statins, \% & 15 \\
Insulin, \% & 1.8 \\
Oral antidiabetic drugs, \% & 6.1 \\
Aspirin/clopidogrel, \% & 18 \\
\hline & \\
\hline
\end{tabular}

The majority had regular medication (70\%). Almost half the cohort reported some type of cardiovascular medication (45\%), with antihypertensive medication being the most prevalent (32\%). Fifteen percent reported use of statins, while insulin and oral antiglycemic drugs were reported in 2 and 6\%, respectively. After excluding individuals with known diabetes mellitus and individuals with a fasting plasma glucose $\geq 7.0 \mathrm{mmol} / \mathrm{l}$, the study population consisted of 459 females and 438 males. 51 females and 88 males had a history of CVD (myocardial infarction, stroke and heart failure, but not hypertension).

\section{Mann-Whitney Tests Comparing Individuals with and without CVD}

For females, only transferrin, triglycerides and urate showed significant differences between the groups at the $\mathrm{p}=0.05$ level. For males, albumin, apolipoprotein A1, apolipoprotein B, total cholesterol, HDL-cholesterol,
LDL-cholesterol, calcium, creatinine, urate and urea showed significant differences between the groups.

\section{Reference Values}

The 2.5th and 97.5th percentiles for each subgroup are presented in table 4 together with the corresponding values according to NORIP [8].

Reference values calculated from the whole population and a subpopulation without CVD showed strong concordance. Only the lower limit of the reference values for cholesterol and LDL-cholesterol for all men were outside the $90 \%$ CI for males without CVD.

In the present study, the upper reference limit for alanine aminotransferase in men was significantly lower than the values reported in NORIP [8]. The lower reference values for albumin and alkaline phosphatase were slightly lower, while the upper reference interval for amylase was below the $90 \%$ CI reported in NORIP. The upper reference interval for bilirubin was lower, while the upper reference interval for calcium was higher. In total, 5.3\% of the samples had values over 2.60 , and $10.2 \%$ of the values were higher than the upper reference limit in NORIP $(2.51 \mathrm{mmol} / \mathrm{l})$. We also found lower creatinine values than in NORIP, which could indicate lower muscle mass or better kidney function in our cohort. Differences for the upper limits of the reference intervals for lactate dehydrogenase, sodium and urate were observed as well. Thus, the reference values obtained in the present study differed from NORIP for several of the studied analytes.

\section{Discussion}

Setting up reference values representative of local service populations is a major responsibility for clinical laboratories. Such reference values often contain few or no individuals older than 65 years of age. Following the recommendations of the International Federation of Clinical Chemistry, we have established reference values based on 70-year-old healthy males and females for a group of common serum analytes. The study population was recruited in the county of Uppland, Sweden. Diabetes and elevated fasting plasma glucose were used as exclusion criteria for the study. Elderly individuals often have illnesses or are on some type of medication. The number of individuals with CVD or taking cardiovascular medication increases with age, and the prevalence in certain elderly populations may be very high. In this population, $70 \%$ used medication regularly and almost half the population used cardiovascular drugs. If such individuals are excluded, the end result may 
Table 4. Calculated upper (97.5th percentile) and lower (2.5th percentile) limits for the reference interval and $90 \%$ CI (in brackets)

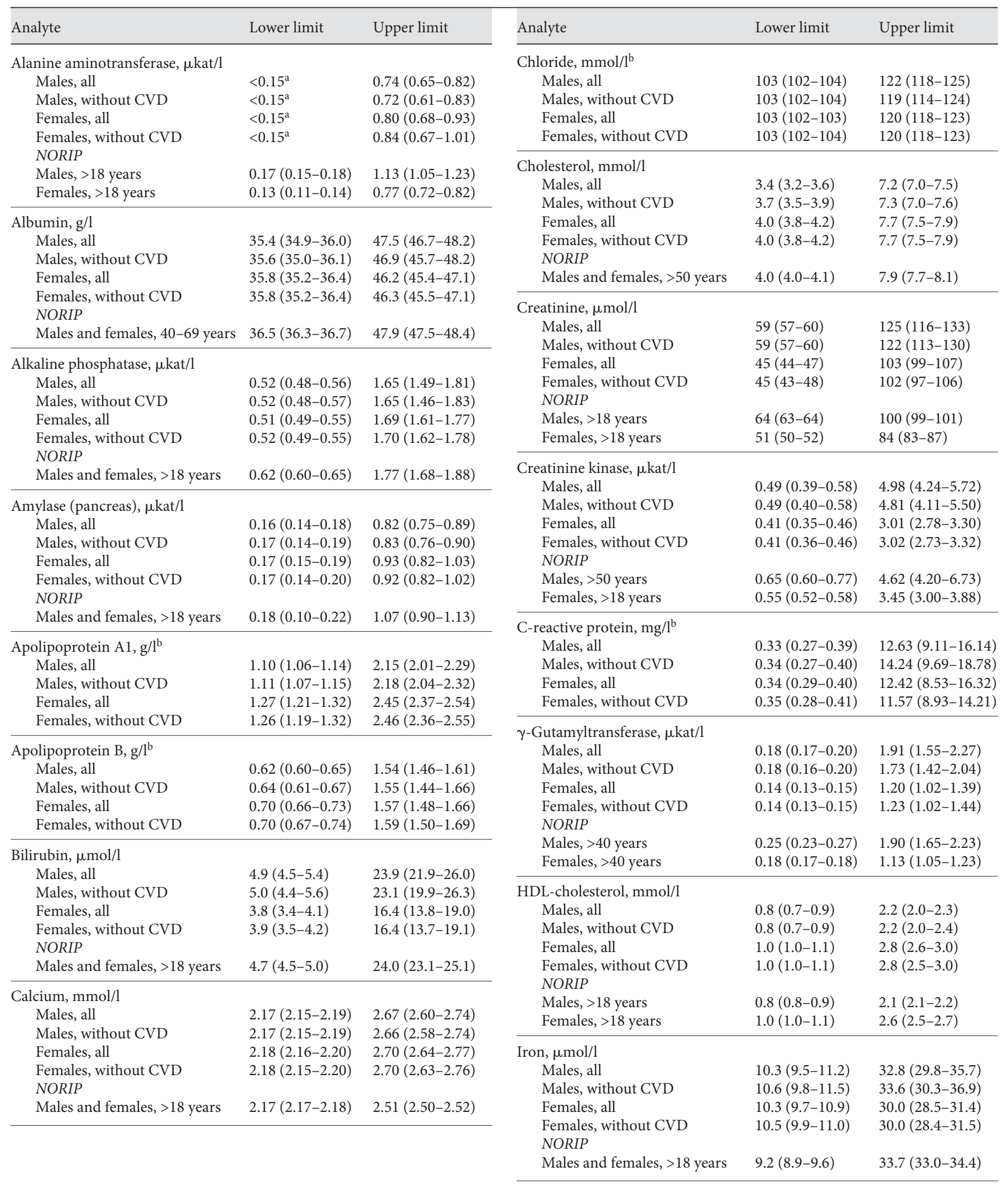


Table 4 (continued)

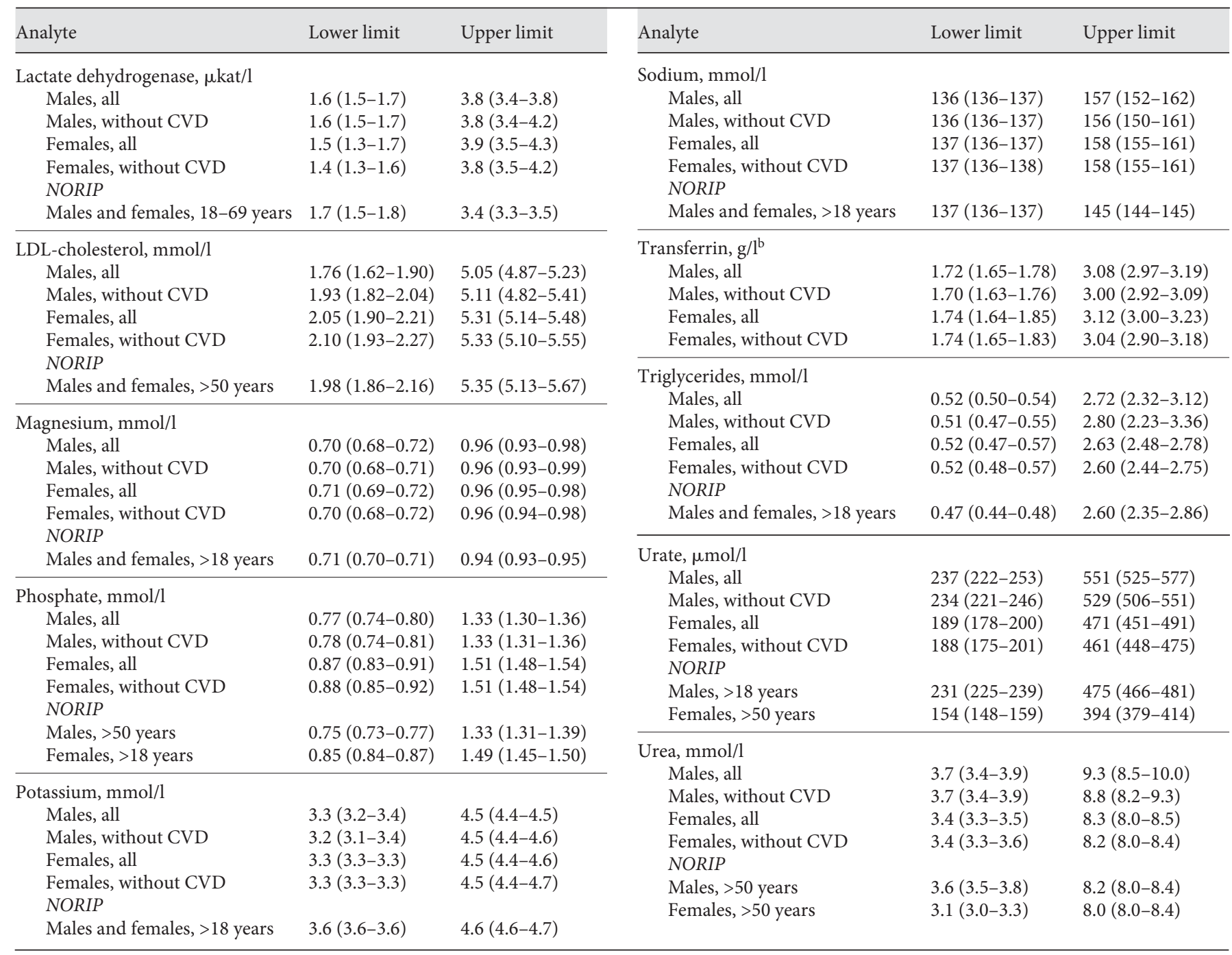

${ }^{a}$ Denotes that the $90 \%$ CI could not be calculated as the instrument did not report ALT values $<0.15 \mu$ kat $/ 1$, and several test results were $<0.15$. NORIP 2000 recommended reference intervals are provided for comparison.

${ }^{\mathrm{b}}$ Not determined in NORIP.

be a very small subpopulation. One could argue that such a small subpopulation is not representative of an elderly population. We thus decided to only exclude patients with diabetes and elevated fasting glucose, as most elderly populations in the study groups contain several individuals with a history of CVD or use of cardiovascular medication. Mann-Whitney tests comparing the groups with and without CVD showed that there were few significant differences between the 2 groups. However, even if there were significant differences between the groups, the $90 \%$ CI for the reference values was not separated.
For males, albumin, apolipoprotein A1, apolipoprotein B, cholesterol, HDL-cholesterol, LDL-cholesterol, calcium, creatinine, urate and urea showed significant differences between the total population and the group without CVD. However, only the lower limit of the lower reference values for cholesterol and LDL-cholesterol for all men were outside the $90 \%$ CI for males without CVD. The lower reference values for these analytes are usually of less importance than the upper reference values for clinical decisions. 
The reference values with and without the CVD group are presented in table 4 . We chose to divide the reference values according to sex, as most laboratory information systems handle sex-specific reference values. For several of the analytes, the sex differences are so small that the same reference interval could be used for both sexes. The reference values were compared with the corresponding values obtained in NORIP (presented in table 4) [8], and there were clear differences. In many cases, such differences are difficult to explain. The NORIP results increased the upper reference interval for alanine aminotransferase in men in most Swedish laboratories, while the pre-NORIP upper reference interval was similar to the one found in the present study. This could indicate that the NORIP limit is a bit high. NORIP also resulted in a lowering of the reference intervals for calcium [11]. There may be some unknown cases of hyperparathyroidism in our study group, but the number of individuals with increased calcium levels is too large to be explained by hyperparathyroidism alone. Thus, it seems that the NORIP reference interval for calcium is too low for this age group. NORIP had very rigorous criteria for blood sampling in comparison with clinical praxis [12]. This may have resulted in a bias, as stasis and similar methods are known to increase calcium values. How- ever, there may also be a true difference as an earlier study reports approximately $0.05 \mathrm{mmol} / \mathrm{l}$ higher calcium in individuals $>60$ years [11]. The lower creatinine values in the present study are most likely due to the reduced muscle mass that occurs in elderly individuals.

The present sample is limited to Caucasians aged 70 years. Caution should therefore be made in drawing conclusions for other ethnic and age groups. The methods used in this study are specified in table 2 . As the choice of method may influence the test results, the results in this study may not be representative for other types of methods.

In our opinion, reference values should be re-evaluated regularly (e.g. every 10 years) to ensure that the reference values are still appropriate for the patients of today. Sex- and age-specific reference values should be used to interpret results of laboratory screening tests.

\section{Acknowledgement}

This study was financially supported by the Uppsala University Hospital Research Fund.

\section{References}

1 Mathias D, Gebhard J, Wilhelm L, SchmidtGayk H, Mathias I: 26 years of external quality controls for the screening of congenital metabolic disorders in newborns - a summary of the results. Clin Lab 2008;54:103108.

- 2 Fuentes-Arderiu X, Mas-Serra R, AlumàTrullàs A, Mart-Marcet MI, Dot-Bach D; Catalan Association for Clinical Laboratory Sciences: Guideline for the production of multicentre physiological reference values using the same measurement system. A proposal of the Catalan Association for Clinical Laboratory Sciences. Clin Chem Lab Med 2004;42:778-782.

3 Rustad P, Felding P, Lahti A: Proposal for guidelines to establish common biological reference intervals in large geographical areas for biochemical quantities measured frequently in serum and plasma. Clin Chem Lab Med 2004;42:783-791.

-4 Guidi GC, Lippi G, Solero GP, Poli G, Plebani M: Managing transferability of laboratory data. Clin Chim Acta 2006;374:57-62.
5 Ferre-Masferrer M, Fuentes-Arderiu X, Puchal-Ane R: Indirect reference limits estimated from patients' results by three mathematical procedures. Clin Chim Acta 1999; 279:97-105.

6 6 Lind L, Fors N, Hall J, Marttala K, Stenborg A: A comparison of three different methods to evaluate endothelium-dependent vasodilation in the elderly: the Prospective Investigation of the Vasculature in Uppsala Seniors (PIVUS) study. Arterioscler Thromb Vasc Biol 2005;25:2368-2375.

7 Bergmeyer HU, Hørder M, Rej R: International Federation of Clinical Chemistry (IFCC) Scientific Committee, Analytical Section: approved recommendation (1985) on IFCC methods for the measurement of catalytic concentration of enzymes. Part 3. IFCC method for alanine aminotransferase (L-alanine: 2-oxoglutarate aminotransferase, EC 2.6.1.2). J Clin Chem Clin Biochem 1986;24:481-495. $\checkmark 8$ Rustad P, Felding P, Franzson L, Kairisto V Lahti A, Martensson A, Hyltoft PP, Simonsson P, Steensland H, Uldall A: The Nordic Reference Interval Project 2000: recommended reference intervals for 25 common biochemical properties. Scand J Clin Lab Invest 2004;64:271-284.

-9 Solberg HE: RefVal: a program implementing the recommendations of the International Federation of Clinical Chemistry on the statistical treatment of reference values. Comput Methods Programs Biomed 1995; 48:247-256.

10 Solberg HE, Grasbeck R: Reference values.

Adv Clin Chem 1989;27:1-79.
Rustad P, Felding P, Lahti A, Hyltoft Petersen $P$ : Descriptive analytical data and consequences for calculation of common reference intervals in the Nordic Reference Interval Project 2000. Scand J Clin Lab Invest 2004; 64:343-370.

12 Felding $\mathrm{P}$, Rustad $\mathrm{P}$, Mårtensson A, Kairisto V, Franzson L, Hyltoft Petersen P, Uldall A: Reference individuals, blood collection, treatment of samples and descriptive data from the questionnaire in the Nordic Reference Interval Project 2000. Scand J Clin Lab Invest 2004;64:327-342. 